(C) [2001] IEEE. Reprinted, with permission, from [W.S. Wijesoma K.R.S. Kodagoda A.

P. Balasuriya E.K.Teoh, Road Edge and Lane Boundary Detection using Laser and Vision, Prowdings of the 2001 IEEEIRsJ International Conference on Intelligent Robots and Systems, Nov. 2001]. This material is posted here with permission of the IEEE. Such permission of the IEEE does not in any way imply IEEE endorsement of any of the University of Technology, Sydney's products or services. Internal or personal use of this material is permitted. However, permission to reprint/republish this material for advertising or promotional purposes or for creating new collective works for resale or redistribution must be obtained from the IEEE by writing to pubspermissions@ieee.org. By choosing to view this document, you agree to all provisions of the copyright laws protecting it 


\title{
Road Edge and Lane Boundary Detection using Laser and Vision
}

\author{
W.S. Wijesoma K.R.S. Kodagoda A. P. Balasuriya E.K.Teoh \\ Department of Electrical and Electronic Engineering \\ Nanyang Technological University, \\ Singapore639798. \\ e-mail: eswwijesoma@ntu.edu.sg
}

\begin{abstract}
This paper presents a methodology for extracting road edge and lane information for smart and intelligent navigation of vehicles. The range information provided by a fast laser range-measuring device is processed by an extended Kalman filter to extract the road edge or curb information. The resultant road edge information is used to aid in the extraction of the lane boundary from a CCD camera image. Hough Transform (HT) is used to extract the candidate lane boundary edges, and the most probable lane boundary is determined using an Active Line Model based on minimizing an appropriate Energy function. Experimental results are presented to demonstrate the effectiveness of the combined Laser and Vision strategy for road-edge and lane boundary detection.
\end{abstract}

\section{Introduction}

Automatic road edge and lane boundary detection are necessary prerequisites for intelligent autonomous and smart vehicle applications. In the application of forward collision avoidance road edges and lane boundaries help to distinguish potential collision threats in terms of their relevance to the intended path of the vehicle. Pavement and mid-line of the road can be further used in local navigation, obstacle avoidance and localization of mobile robots or autonomous guided vehicles (AGV). Apart from such applications automatic lane detection can provide assistance to human drivers. One such application can be in drowsy driver warning systems. The knowledge of the lane boundaries relative to the vehicle enables a driver assistant system to alert a driver as to whether the vehicle is potentially steering off course.

Traditionally lane detection techniques have relied on images captured from CCD cameras. The use of intensity gradient information of a visual image to detect lanes is common as evident from the literature. Many gradient-based detection algorithms which are used to detect lane and pavement boundaries, apply a threshold to the image gradient magnitude $[1,2]$. The performance of such gradient-based edge detection algorithms are good if the images have uniform regions with good separation between regions. However, real road, lighting and weather conditions seldom give rise to such clear and contrasting images. Of recent, the use of mm-wave Radar images as an alternative to CCD Vision images for lane detection has received some interest $[3,4,5]$. The major advantage gained in using mm-wave radar is the variety of conditions under which the system can yield acceptable performance. The shortcomings of using mm-wave radar imagery are the higher costs, complexity of the algorithms in the face of backscatter from off-road structures, and large power requirements.

In this paper a methodology is proposed using both laser and vision for detection of road edges and lane boundaries effectively. A laser range measurement system can provide a low cost alternative to depth or range measurement under poor lighting, visibility and bad weather conditions [6]. Laser range finders have found applications in autonomous navigation systems. But their uses have been mainly in obstacle detection, navigation [7] and in some cases localization [8]. In our application, laser is used mainly to detect the road edges, and hence subsequently, assist the vision system to detect the lane boundaries, especially the middle line of the road. In this respect the technique proposed is novel. The 
rest of this paper is organized as follows. In Section 2, the detection of road edges is formulated as an Extended Kalman Filtering (EKF) problem. In Section 3, The Hough Transform (HT) with Active Line Model (ALM) approach is used to find the most prominent mid-line or the right lane boundary. Experimental results are presented and discussed in Section 4. Section 5 concludes the paper.

\section{EKF for road edge detection}

The SICK planar two dimensional (2D) laser range measurement system (LMS) [6] is mounted on the mobile platform looking down on the road at an angle as shown in Figure 2. The LMS scans a laser spot beam from right to left on a plane inclined at an angle of $\alpha_{\mathrm{L}}$ to the road surface. It is assumed that the road is flat and horizontal. The road edges or curbs are assumed to be the vertical surfaces at either side of the road. The pavements at a higher elevation and parallel to the road are also assumed to be horizontal and flat. Under the above assumptions, a single planar sweep of the laser spot beam will yield different sets of range data points corresponding to the pavement surfaces on either side of the road, road edge surfaces, and the road surface. Each of the set of points, under ideal and assumed conditions, defines a straight-line segment since the intersection of two planes (laser scanning plane and the external surface plane) give rise to a line. Thus, it may be noted that the road edges correspond to points of intersection of the line segments or the points at which there are significant discontinuity or gradient change of the path trace of the laser beam. In practice measurement noise and other sources of noise (specula reflections) can corrupt the data points thus making it difficult to detect the points of discontinuity. The problem of noise filtering and simultaneous detection of the points of discontinuity are handled by an extended Kalman filter. For a given region (e.g. road, edge surface or pavement surface) the evolution of the range data, $\left(d_{i}\right)$, provided by the LMS can be described by an appropriate process model. The model is so formulated to predict the next range data $\left(d_{i+1}\right)$ given the past two measurements $\left(d_{i-1}, d_{i}\right)$ since the points evolve on a straight line. This process model is combined with the LMS range data using a Kalman filter to obtain a filtered estimate of the range measurements. However, the filtering is effective and valid provided that the model of the process adequately describes the evolution of the range data points. This is true for data lying on the same region. However, the prediction would be very different from the measured data at the boundary separating the two regions, as the process models for the two regions are different, although of they are of the same structure. This prediction error is appropriately thresholded to obtain a candidate point of discontinuity or edge.

Consider the three points $\mathrm{P} 1, \mathrm{P} 2$ and $\mathrm{P} 3$, lying on a line segment $\mathrm{L} 1$, at a range of $d_{i}, d_{i+1}$, and $d_{i+2}$ respectively, as shown in Figure 1. Using elementary trigonometry it can be shown that their relationship is as follows:

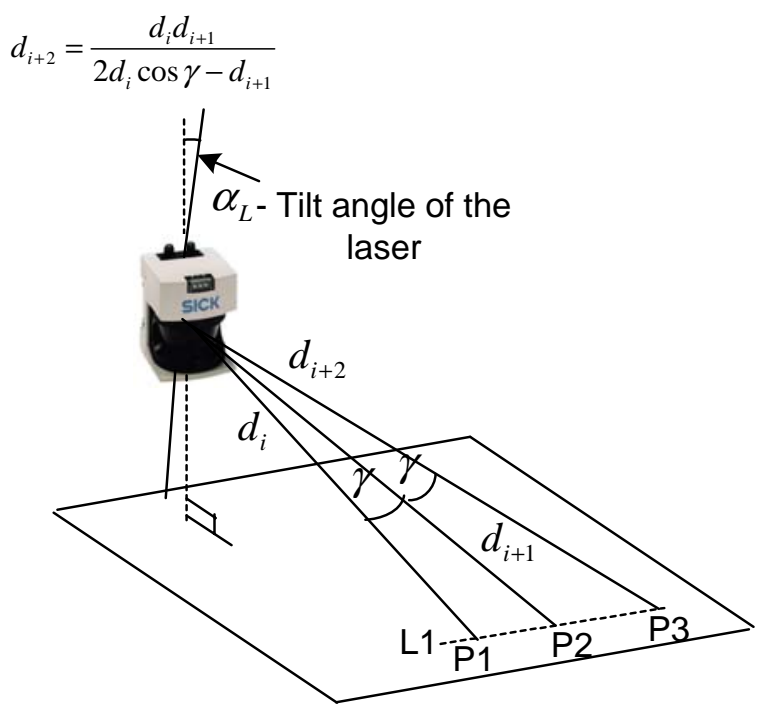

Figure 1. Three consecutive data points on a road surface

Now we setup up the process model by choosing the state variable $x_{1}, x_{2}$ as follows:

$x_{1}(k+1)=d_{i+2}$

$x_{2}(k+1)=x_{1}(k)=d_{i+1}$

where, $x_{1}(k)$ and $x_{2}(k)$ represents the states at the pseudo discrete time index $k$. Hence from equations (1) and (2) we obtain the non-linear process model:

$x_{1}(k+1)=\frac{x_{2}(k) x_{1}(k)}{2 x_{2}(k) \cos \gamma-x_{1}(k)}$
$x_{2}(k+1)=x_{1}(k)$ 
Further, it can be represented in the vector form as, $\underset{\sim}{x}(k+1)=\underset{\sim}{F}(\underset{\sim}{x}(k), \underset{\sim}{u}(k), \underset{\sim}{w}(k))$

where, $\underset{\sim}{u}(k)$ and $\underset{\sim}{w}(k)$ are input to the plant and process noise respectively. The process is assumed deterministic and hence the process noise covariance, $\mathrm{Q}$, is zero. And also, in this case there is no input. Since the process model is non-linear we apply the extended Kalman filter algorithm. Now, the nonlinear function $\boldsymbol{F ( . )}$ in equation (4) can be expanded as a Taylor series about the previous prediction, $\hat{x}(k / k)$.

$A_{i, j]}=\frac{\partial F_{[i]}}{\partial x_{[j]}}(\hat{x}(k), 0,0)$

$\boldsymbol{A}$ is the Jacobian matrix of partial derivatives of $\boldsymbol{F}($.) with respect to $x$, which can be used to form a relationship between small perturbations of the state, yielding the linearized model:

$\delta \underset{\sim}{x}(k+1) \approx \underset{\sim}{A} \delta \underset{\sim}{x}(k)$

where,

$$
A_{11}=\frac{2 x_{2}^{2}(k) \cos \gamma}{\left(2 x_{2}(k) \cos \gamma-x_{1}(k)\right)^{2}}, \quad A_{21}=1
$$

$$
A_{12}=\frac{-x_{1}^{2}(k)}{\left(2 x_{2}(k) \cos \gamma-x_{1}(k)\right)^{2}}, \quad A_{22}=0
$$

$\underset{\sim}{A}$ matrix is to be re-evaluated at every new time step $k$.

The range measurement equation is :

$$
\begin{aligned}
& z(k)=d_{i+1}=\left[\begin{array}{ll}
1 & 0
\end{array}\right]\left[\begin{array}{c}
x_{1}(k) \\
x_{2}(k)
\end{array}\right]+v(k) \\
& z(k)=\mathbf{C} \underset{\sim}{x}(k)+v(k)
\end{aligned}
$$

where, $\boldsymbol{C}$ is the observation matrix, and $v(k)$ is the measurement noise with known variance $\sigma_{r}^{2}$. Now, the Extended Kalman Filter (EKF) algorithm as detailed in [9] is used to detect the pavement edges.

1. Filter Initialization: A reasonable initialization error covariance, $\underset{\sim}{P}(k / k)$ can be estimated using two range measurements, at time $k=1$ and $k=2$. From equation (7), $\hat{x}_{1}(1)=z(1), \hat{x}_{1}(2)=z(2)$. From equation $(3), \hat{x}_{2}(2)=\hat{x}_{1}(1)$ and hence,

$$
\underset{\sim}{\hat{x}}(2 / 2)=\left[\begin{array}{c}
z(2) \\
z(1)
\end{array}\right]
$$

Assuming the individual noise samples are uncorrelated,

$$
P(2 / 2)=\left[\begin{array}{cc}
\sigma_{r}^{2}(2) & 0 \\
0 & \sigma_{r}^{2}(1)
\end{array}\right]
$$

2. Filter Prediction: Determine the predicted state and error covariance matrix.

$$
\begin{aligned}
& A_{i i, j]}=\frac{\partial F_{[i]}}{\partial x_{[j]}}(\hat{x}(k), 0,0) \\
& \underset{\sim}{\hat{x}}(k+1 / k)=\underset{\sim}{F}(\underset{\sim}{\hat{\gamma}}(k / k)) \\
& \underset{\sim}{P}(k+1 / k)=\underset{\sim}{P}(k / k){\underset{\sim}{A}}^{T}
\end{aligned}
$$

where, $\underset{\sim}{P}(k+1 / k)$ is the predicted value of the error covariance matrix at time $k+1$, given all observations up to and including time $k$.

3. Innovation : Determine the difference between the observation and the predicted observation.

$\omega(k+1)=z(k+1)-\underset{\sim}{\operatorname{Cx}}(k+1 / k)$

4. Variance of Innovation : Find the variance associated with the innovation.

$s(k+1)=\sigma_{r}^{2}(k+1)+\underset{\sim}{\operatorname{C}}(k+1 / k) C_{\sim}^{T}$

where $\sigma_{r}^{2}(k+1)$ denotes the range variance at time $k+1$.

\section{Validation gate :}

$D(k+1)=\omega^{2}(k+1) s^{-1}(k+1)$

A observation that falls outside of a threshold value for $D(k+1)$ is defined as possible edges.

6. Define $\underset{\sim}{W}(k+1)::$

$\underset{\sim}{W}(k+1)=\underset{\sim}{P}(k+1 / k){\underset{\sim}{T}}^{T} s^{-1}(k+1)$

\section{State Vector Update :}

$$
\begin{aligned}
& \underset{\sim}{\hat{X}}(k+1 / k+1)=\underset{\sim}{\hat{x}}(k+1 / k)+\underset{\sim}{W}(k+1) \omega(k+1) \\
& \underset{\sim}{P}(k+1 / k+1)=\underset{\sim}{P}(k+1 / k)-\underset{\sim}{W}(k+1) s(k+1) \underset{\sim}{W}(k+1)
\end{aligned}
$$

8. Recalculate A : A must be recalculated, based on the new prediction $\underset{\sim}{\hat{x}}(k+1 / k+1)$ after which, step 2 can be executed to continue the cycle.

\section{HT for Middle lane detection}

The laser scanner and CCD camera are arranged in such 
a way that the laser-road intersection points are within the field of view of the camera (see Figure 2). The detected pavement edge points are then mapped to the image plane.

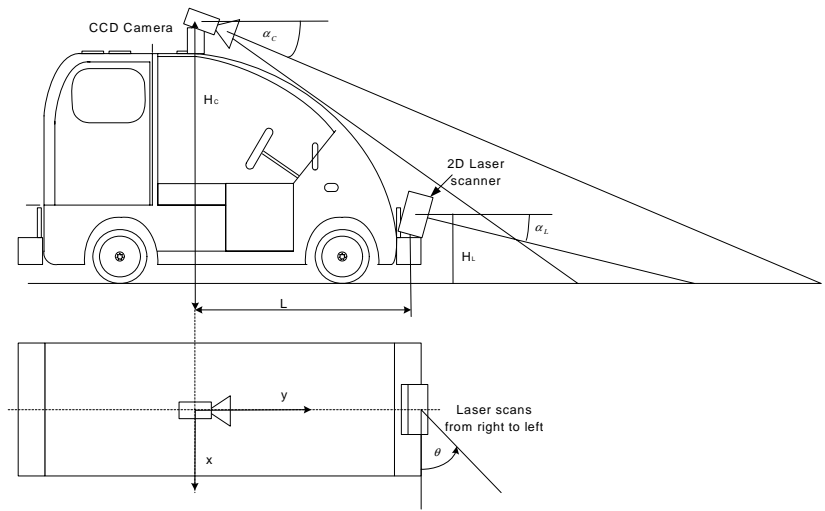

Figure 2 Laser camera arrangement of the mobile robot

Row ( $r$ ) and column (c) coordinates of the detected pavement edge points in the image plane are,

$$
\begin{aligned}
& r=\frac{H_{C}}{\left(d \sin \theta \cos \alpha_{L}+L\right) r f} \\
& c=\frac{d \cos \theta \cos \alpha_{L}}{\left(d \sin \theta \cos \alpha_{L}+L\right) c f}
\end{aligned}
$$

where, $(d, \theta)$ are the laser coordinates, $H_{c}$ is the camera height, $L$ is the horizontal distance between the laser and the camera, $r f$ is the height of a pixel on the focal plane divided by focal length, $c f$ is the width of a pixel on the focal plane divided by the focal length. It is assumed that the road has a middle line. Then using the pavement edge information, the position of the middle line can be determined. A search area width is estimated based on heuristics. Hough Transform (HT) is then performed to find possible lines within that search area (window ' 1 ' of Figure 3). This will drastically reduce the high computational requirements required by the HT. Again a second search area (window ' 2 ' of Figure 3 ) is estimated based on space continuity of lane structure. Then HT is performed within that search area. This procedure is repeated until a predefined window boundary on the image is exceeded.

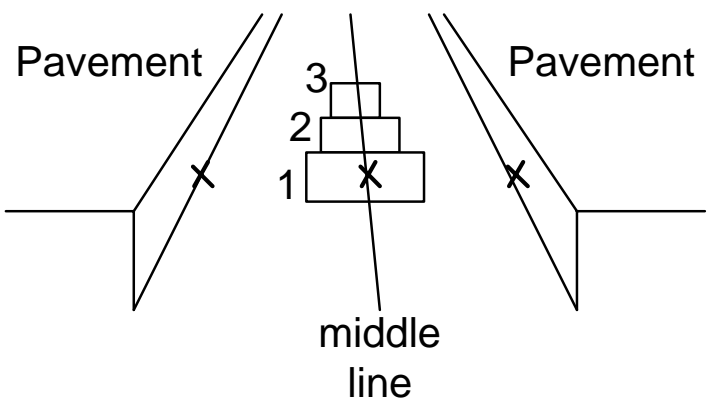

Figure 3 Middle line detection using search areas

After performing HT for each search area, the candidate lines of each search area are extracted. Among all the candidate lines extracted, the most prominent line is determined by the Active Line Model (ALM), which minimizes the following energy:

$$
\begin{aligned}
E= & \alpha .\left|\left(L_{i+1}^{e}-L_{i}^{s}\right)\right| \\
& +\beta \cdot\left|\left[\tan ^{-1}\left(\frac{s r_{i+1}-e r_{i+1}}{s c_{i+1}-e c_{i+1}}\right)-\tan ^{-1}\left(\frac{s r_{i}-e r_{i}}{s c_{i}-e c_{i}}\right)\right]\right|
\end{aligned}
$$

where, $\alpha$ and $\beta$ are weighting factors, superscripts, $e$ and $s$, denote start and end of a line, $L, \quad s r_{i}$ denotes start of the row coordinate of the $i^{\text {th }}$ line, $e c_{i}$ denotes the end of the column coordinate of the $i^{\text {th }}$ line, and so on. Please note that $i^{\text {th }}$ line is from $i^{\text {th }}$ window and so on.

\section{Experimental Results}

Experiments were carried out on the mobile robot test-bed, designed and developed by Nanyang Technological University, Singapore (see Figure 4).

\subsection{Road Edge Detection}

The SICK laser scanner [6] used provides range 2D range data over $180^{\circ}$ span at $1^{\circ}$ intervals. A typical data set resulting from a single sweep or scan is shown in Figure 5. It can be noted that the range data can be highly scattered depending on the environment.

Figure 6 shows the actual data and predicted data by EKF in $d-\theta$ domain (only interested part of the data are shown in order to make the graph clear). The EKF starts at the mid point of the data set and proceed towards both 
ends. " $x$ " denotes the actual data while "*” denote the predicted data by the EKF.

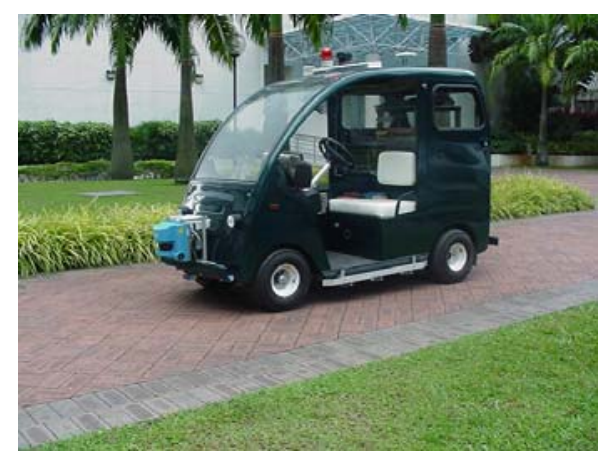

Figure 4 Experimental test-bed AGV

Using a threshold for the validation gate, $D(k+1)$, the edges can be detected (see 'star marks' in Figure 7). However, it is possible to detect false edges due to the spurious data that result from specula reflections. Using the gradient information of predicted data can eliminate such points. There is a positive to negative gradient change in the road to pavement transition point (see the predicted point corresponding to "Detected Left edge" in Figure 6) while on the other side the gradient change is negative to positive (see the predicted point corresponding to "Detected Right edge" in Figure 6). As stated the EKF algorithm starts at $\theta=90^{\circ}$ and proceed towards both sides. Figure 8 shows the actual and predicted data on the ground plane (only interested part of the data are shown).

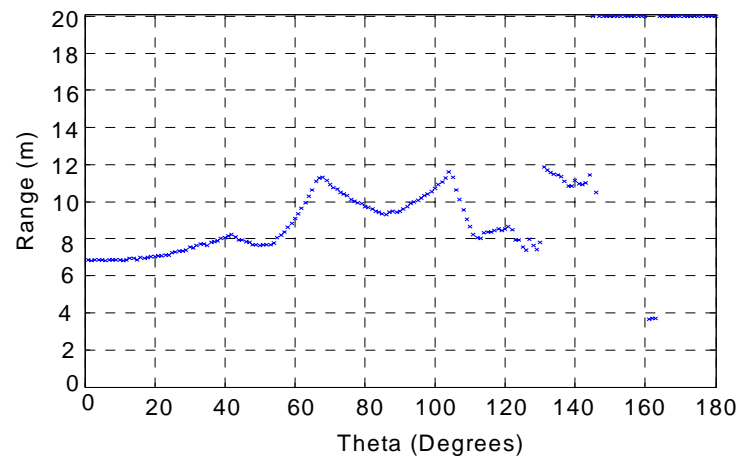

\section{Figure 5 A typical $d-\theta$ data set on a road}

The laser data with edge information can then be mapped on to the image plane. The mapped data is as shown in the Figure 9 and is indicated as ' $x$ ' points. It is to be noted that such points provide the depth information of the corresponding image points.

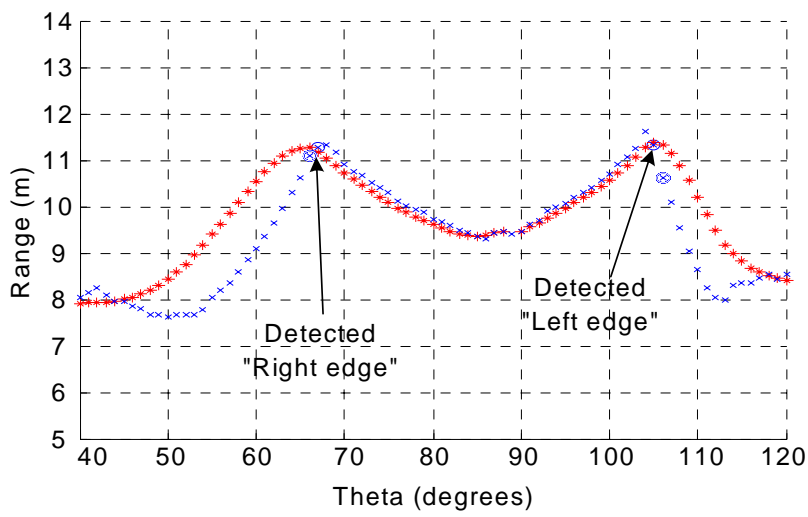

Figure $6 d-\theta$ plot for actual (x) and predicted (*) data

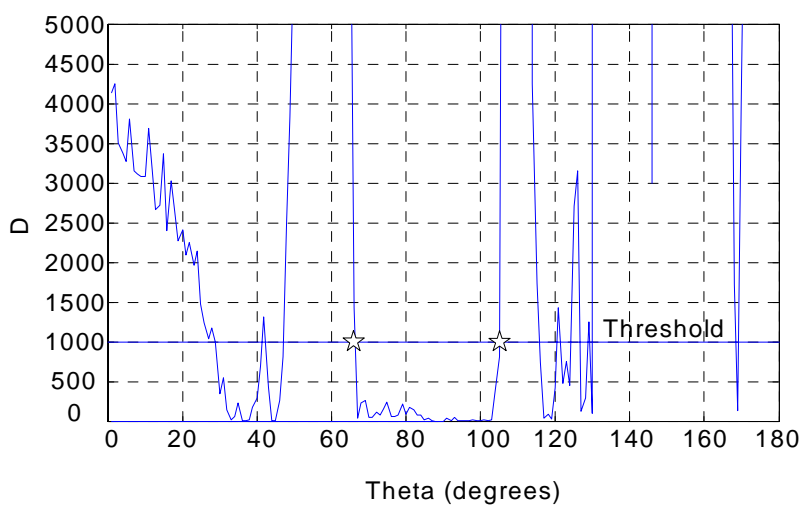

Figure $7 \theta$ vs validation gate, $D(k+1)$

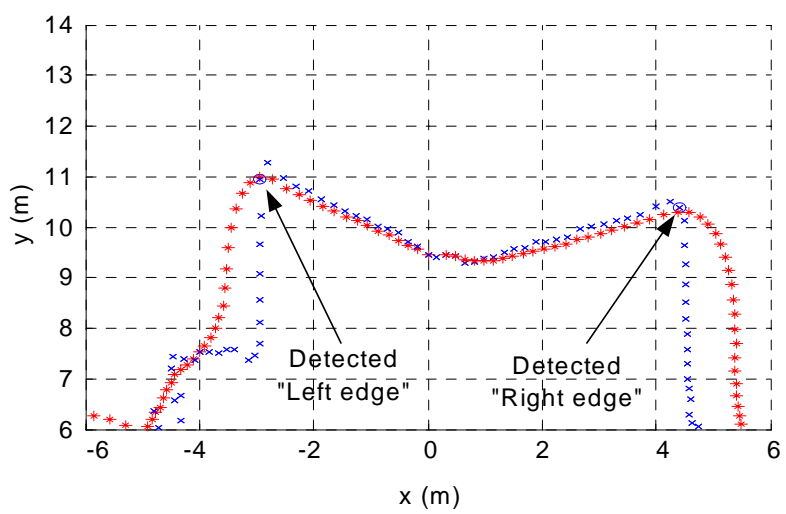

Figure 8 Actual $(\mathrm{x})$ and predicted $\left(^{*}\right)$ data projected on to the ground plane.

\subsection{Mid- lane or lane boundary detection}

Once the search area for mid line is determined, HT is used to find possible edges. Then a second search area width is estimated based on the space continuity of lane structure. HT is used to find possible lines within that 
search area. The same procedure is repeated until it reaches a predefined window. The lines detected using HT in different search areas are shown in Figure 10.

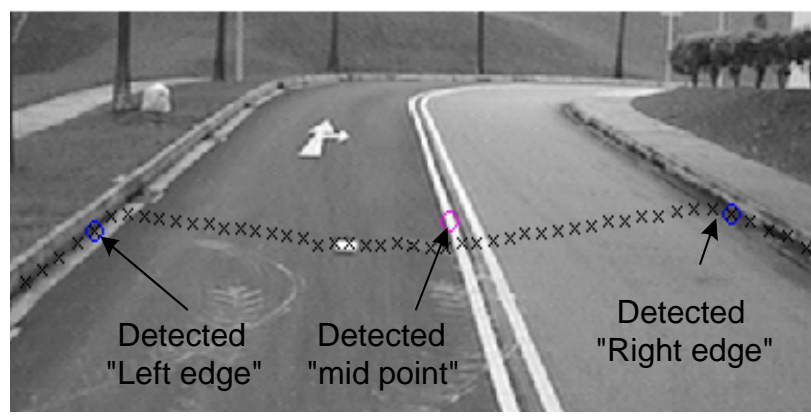

Figure 9 Projected laser data on the image plane

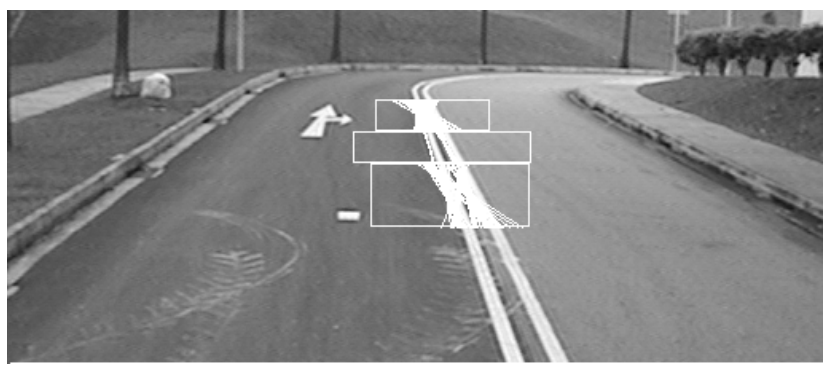

Figure 10 Detected lines in three search areas

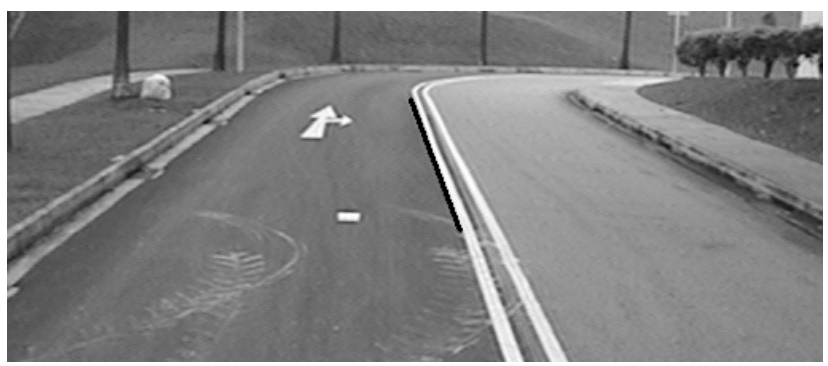

Figure 11 Detected mid-line using HT and ALM

Then the most prominent line is determined by applying the ALM, which is given in section 3. The result is shown in Figure 11. The mid line so detected and the range data of the road edges can be effectively used for navigation and localization.

\section{Conclusions}

Road edge information can be effectively extracted using range information provided by a $2 \mathrm{D}$ laser scanner. The Kalman filter approach enables the detection of the road edges whilst filtering the noisy range data. Such edge information can be incorporated within an image from a CCD camera to determine the lane boundary in a computationally efficient manner. The technique not just provides for faster detection of edges and lane boundaries, but also doing the same under poor visibility conditions. The experiments on actual roads show the robustness of the algorithm. Our future intention is to generalize the camera and laser fusion methodology to detect all possible road edge and lane boundary shapes that can arise in all types of road configurations.

\section{References}

[1] S.K. Kenue, "LANELOK, Detection of Lane Boundaries and Vehicle Tracking Using Image-Processing Techniques", Parts I and II, SPIE Mobile Robots IV, 1989.

[2] J.P. Gonzalez, Umit Ozguner, "Lane Detection using Histogram-Based Segmentation and Decision Trees”, Procs. of IEEE Intelligent Transportation Systems, 2000, pp346-351.

[3] Sridhar Lakshmanan and david Grimmer, "A Deformable Template Approach to Detecting Straight Edges in Radar Images", IEEE Trans. on Pattern Analysis and Machine Intelligence, Vol 18, No 4, 1996, pp438-443.

[4] Bing Ma, Sridhar Lakshmanan and Alfred O. Hero, "Detection of Curved Road Edges in Radar Images Via Deformable Templates", Procs. of IEEE Intl. Conf. Image Processing, 1997, pp857-860.

[5] Bing Ma, Sridhar Lakshmanan and Alfred O. Hero, "Pavement Boundary Detection Via Circular Shape Models", Procs. of IEEE Intelligent Vehicles Symposium, 2000, pp644-649.

[6] Technical Description, SICK AG, Auto Ident, Germany.

[7] D. Dedieu, V. Cadenat and P. Soueres, "Mixed camera-laser based control for mobile robot navigation", Procs. of IEEE/RSJ Int. conf. On Intelligent Robots and Systems, 2000.

[8] Kai O. Arras, Nicola Tomatis and Roland Siegwart, "Multisensor On-the-fly Localization using Laser and Vision”, Procs. of IEEE/RSJ Int. Conf. On Intelligent Robotics and Systems, 2000, pp462-467.

[9] Adams M.D., Sensor Modelling, Design and data processing for Autonomous Navigation, World Scientific Publishing Co. Pte. Ltd, 1999. 